\title{
Alocação de Potência Baseada na Margem de Certeza Equivalente Aplicável a Sistemas sem Fio Limitados por Interferência
}

\section{Power Allocation based on Certain Equivalent Margin Applicable to Wireless Communication Systems Limited by Interference}

\author{
Tadeu Junior Gross ${ }^{1}$; Taufik Abrão ${ }^{2}$; Paul Jean Etienne Jeszensky ${ }^{3}$
}

\begin{abstract}
Resumo
Neste artigo analisa-se o método de alocação de potência para redes de comunicação sem fio, limitadas em interferência de múltiplo acesso e com desvanecimento Rayleigh. O método leva em consideração a variação estatística da potência do sinal desejado e dos sinais interferentes e aloca ótima e dinamicamente os recursos de potência disponíveis no sistema. Esta alocação é feita levando-se em consideração as restrições impostas pela probabilidade de ocorrer falha na comunicação (interrupção) para cada par transmissor/receptor. Baseados na teoria de autovalor de Perron-Frobenius e na teoria de programação geométrica (GP), foram encontrados alguns resultados para o problema de otimização em questão. Um GP é um tipo especial de problema de otimização que pode ser transformado em um problema de otimização não linear convexo pela mudança de variáveis e assim ser resolvido globalmente. Também foi apresentado um método iterativo de rápida convergência para encontrar a alocação de potência ótima visando minimizar a probabilidade de falha na comunicação.
\end{abstract}

Palavras-chave: DS-CDMA. Algoritmo para Controle de Potência. Programação Geométrica. Teoria do Autovalor de Perron-Frobenius.

\begin{abstract}
This paper analyzes the method of power allocation for wireless communication networks, limited by multiple access interference under fading Rayleigh channels. The method takes into account the statistical variation of the desired and interfering signals power and optimally and dynamically allocates the power resources in the system. This allocation is made being taken into account the restrictions imposed by the outage probability for each receiver/transmitter pair. Based on the Perron-Frobenius eigenvalue and geometric programming (GP) theories, some interesting results were found for the optimization problem analyzed. A GP is a special type of optimization problem that can be transformed in a non-linear convex optimization problem simply by the change of variables, and then to be globally solved. Also, an iterative method for fast convergence was shown to find the optimally power allocation seeking to minimize the outage probability in the communication.
\end{abstract}

Keywords: DS-CDMA. Power Control Algorithm. Geometric Programming. Perron-Frobenius Eigenvalue Theory.

\footnotetext{
${ }^{1}$ Perito Criminal Oficial da Polícia Científica (POLITEC-MT), área de Processamento Forense de Sinais Analógicos e Digitais de Áudio, Imagem e Vídeo, tadeu.gross@gmail.com

${ }^{2}$ Docente do Departamento de Engenharia Elétrica da Universidade Estadual de Londrina; taufik@uel.br

${ }^{3}$ Docente do Departamento de Telecomunicações e Controle da Escola Politécnica da USP, pjj@usp.br
} 


\section{Introdução}

Alocação de potência é um fator importante em redes de comunicação sem fio, tendo em vista o aumento na autonomia da bateria do dispositivo móvel, a qual também afeta a utilização racional do espectro de frequência em sistemas de múltiplo acesso. Devido a estes benefícios, este assunto tem sido muito estudado (GOLDSMITH; GREENSTEIN; FOSCHINI, 1994),

(MITRA, 1993), (ANDERSIN; ROSBERG; ZANDER, 1996), (YATES; HUANG, 1995), (YATES, 1995),

( B A M B O S, 1998), (BAMBOS; CHEN; POTTIE, 1995). Esquemas de controle de potência tradicionais, centralizados (ARIYAVISITAKUL, 1992), (ALAVI; NETTLETON, 1983) ，(AEIN, 1973) ou distribuídos (BAMBOS; CHEN; POTTIE, 1995), (FOSCHINI; MILJANIC, 1993), (FOSCHINI; MILJANIC, 1995), assumem que os canais com desvanecimento são quase estacionários. Estes esquemas estão baseados na razão sinal-interferência (SIR) observada no receptor (método distribuído) ou no conhecimento dos ganhos de todos os links (método centralizado). Assim, a hipótese implícita feita é que a atualização no controle de potência é realizada toda vez que o desvanecimento demonstra mudanças no canal, ou seja, sempre que o ganho de qualquer link mude. Em canais de comunicação sem fio, os quais exibem desvanecimento rápido (o desvanecimento pode mudar em intervalos de tempo da ordem de unidades de milisegundos, para $2 \mathrm{GHz}$ e móvel trafegando a $80 \mathrm{~km} / \mathrm{h}$ ), isto pode não ser prático sempre. Atualizações de potência muito frequentes aumentam a complexidade exigida no processamento de sinal.

Em (KANDUKURI; BOYD, 2002) os autores propõem um esquema de controle de potência no qual a potência não precisa ser atualizada sempre que o canal mudar de um estado desvanecido para o outro. Porém, leva-se em conta a variação estatística da SIR de cada par trasmissor-receptor, alocando-se otimamente a potência, com o objetivo de minimizar a probabilidade de interrupção da comunicação (outage probability) induzida pelo desvanecimento, a qual ocorre quando a SIR fica abaixo de um limite inferior SIR ${ }^{\text {th }}$. Desta forma, assume-se que entre sucessivas atualizações de controle de potência, a "quebra" da comunicação ocorre devido ao desvanecimento rápido (do sinal e da interferência) e que fatores como sombreamento log-normal e atenuação dependente da distância permanecem constantes.

Em (KANDUKURI; BOYD, 2002) foi sugerida uma solução global para este problema (de mínima probabilidade de interrupção), mostrando que o mesmo pode ser apresentado como um problema de otimização não linear (NLP) convexo. Em um NLP convexo pode-se garantir que o mínimo encontrado é a solução ótima do problema. A solução ótima para o problema permite que as atualizações de potência sejam realizadas em intervalos de tempo maiores que o intervalo entre dois desvanecimentos Rayleigh sucessivos (como encontrado na literatura até então (GOLDSMITH; GREENSTEIN; FOSCHINI, 1994), (MITRA, 1993), (ANDERSIN; ROSBERG; ZANDER, 1996), (YATES; HUANG, 1995), (YATES, 1995), (BAMBOS, 1998), (BAMBOS; CHEN; POTTIE, 1995), (ARIYAVISITAKUL, 1992), (ALAVI; NETTLETON, 1983), (AEIN, 1973), (FOSCHINI; MILJANIC, 1993), (FOSCHINI; MILJANIC, 1995), (ZANDER, 1992). Tipicamente, estas atualizações podem ser realizadas em intervalos de tempo da ordem de ocorrência das variações do sombreamento log-normal.

A probabilidade de interrupção pode ser reduzida pela alocação de potência, de maneira que cada móvel tenha uma margem extra de SIR, isto é, sua SIR está um pouco acima do valor mínimo SIR $^{\text {th }}$ solicitado para a recepção. Um incremento na margem de SIR reduz a probabilidade, porém custa potência extra. O método dá a cada móvel uma margem de SIR que é diretamente baseada na probabilidade de interrupção induzida pelo desvanecimento. 
Maximizar a mínima SIR presente no sistema, uma aproximação conhecida como SIR balanceada, é um tópico que tem sido bastante estudado (ALAVI; NETTLETON, 1983), (AEIN, 1973), (ZANDER, 1992). Este trabalho, inspirado em (KANDUKURI; BOYD, 2002), estabelece análises detalhadas das relações existentes entre margem de $\mathrm{SIR}^{1}$ e a probabilidade de interrupção ${ }^{2}$ em sistemas de comunicação sem fio limitados por interferência de múltiplo acesso, i.e., sistemas de múltiplo acesso por divisão de código do tipo sequência direta (DS/ CDMA - direct sequence code division multiple access). Foram obtidos os limites superior e inferior da probabilidade de interrupção de acordo com uma dada margem de SIR. Mostrou-se que estes limites são muito estreitos para margens de SIR elevadas. Também são apresentados resultados analíticos sobre a probabilidade de interrupção obtidos pelo esquema de controle de potência desenvolvido para maximizar a margem de SIR. O método de alocação de potência maximizando a margem de SIR tem sido bem estudado (YATES, 1995), ( B A M B O S , 1998), (AEIN, 1973), (FOSCHINI; MILJANIC, 1993), (ZANDER, 1992); porém, são raros na literatura métodos para minimizar a probabilidade de interrupção. Baseado nos resultados de (KANDUKURI; BOYD, 2002), analisa-se aqui dois métodos para minimizar a probabilidade de interrupção. O primeiro encontra o ótimo global, ainda que outras restrições sejam incluídas no problema. O segundo é um método iterativo de rápida convergência, baseado na teoria de PerronFrobenius. Finalmente, expressa-se analiticamente a probabilidade de interrupção através do método de alocação de potência maximizando a margem de SIR, sendo verificado que o resultado é muito próximo da probabilidade de interrupção ótima.

\section{Canal com Desvanecimento}

Considerou-se um sistema SISO com $n$ transmissores e $n$ receptores, identificados por $1, \ldots, n$, com cada transmissor transmitindo com nível de potência $P_{1}, \ldots, P_{n}$, as quais são as variáveis do problema de otimização em questão. O sinal de interesse para o receptor $i$ é o sinal transmitido pelo transmissor $i$. Entenda-se por receptor e transmissor não necessariamente diferentes transmissores e receptores físicos; diferentes receptores, por exemplo, podem fazer referência ao mesmo receptor físico, porém com diferentes canais em frequência, códigos ou feixes em uma antena. A potência recebida do transmissor $j$ para o receptor $i$ é dada por:

$$
P_{i}^{\mathrm{rX}}=G_{i j} F_{i j} P_{j}
$$

O número $G_{i j}$, que é positivo, representa o ganho no percurso (sem incluir desvanecimento) do j-ésimo transmissor para o i-ésimo receptor. O termo ganho pode ser interpretado de diversas maneiras: pode representar a atenuação de potência dependente da distância entre transmissor e receptor, sombreamento log-normal, correlações cruzadas entre códigos em um sistema de acesso múltiplo por divisão de código, CDMA (code division multiple access), bem como ganho dependente da direção da antena. $\mathrm{Na}$ análise abaixo, assume-se que os ganhos $G_{i j}$ sejam constantes com o tempo. Esta aproximação é válida para uma escala no tempo sobre a qual os fatores que determinam $G_{i j}$ são aproximadamente constantes: a distância entre transmissores e receptores, o sombreamento lognormal e o ganho dependente da direção da antena não mudam muito.

Os números $F_{i j}$ modelam o desvanecimento Rayleigh. Assume-se que $F_{i j}$ sejam variáveis aleatórias independentes, com distribuição exponencial e média unitária (STUBER, 1997). Assim, a potência recebida para o receptor $j$ do

${ }^{1}$ Para um esquema de controle de potência projetado para condições quase estacionárias.

${ }^{2}$ Para o mesmo esquema de controle de potência operando em condições de desvanecimento. 
transmissor $j$ é uma variável aleatória distribuída exponencialmente com valor médio dado por:

$$
P_{i}^{\mathrm{rx}}=\mathbb{E}\left[G_{i j} F_{i j} P_{j}\right]=G_{i j} P_{j}
$$

Sendo $\mathbb{E}[\cdot]$ o operador esperança estatística. Faz-se referência a situação onde tanto o sinal desejado quanto os sinais interferentes estão sujeitos ao desvanecimento Rayleigh, como ambiente com desvanecimento Rayleigh/Rayleigh. A hipótese para o ambiente Rayleigh/Rayleigh é que não há linha de visada entre o receptor e o transmissor do sinal de interesse e entre o receptor e os transmissores dos sinais interferentes.

Assume-se também, a hipótese de que a interferência de outros transmissores é muito maior que o ruído branco nos receptores. Assim o ruído branco pode ser ignorado nesta análise. Tanto a hipótese de ambiente Rayleigh/Rayleigh quanto a hipótese de comunicação limitada em interferência são bastante realistas em ambientes urbanos de comunicação sem fio. Em alguns casos, a componente de ruído na SIR pode surgir não apenas do ruído branco no receptor, mas também de usuários co-canal que não estão incluídos na formulação do problema de controle de potência. Isto pode significar que neste caso o ruído não pode ser negligenciado. Uma possível maneira de levar em consideração este fato é assumir que existem mais que $n$ transmissores e que o transmissor $(n+1)$ transmite com potência $P_{n+1}$.

\section{Probabilidade de Interrupção e Margem de Certeza Equivalente}

\section{SIR e Probabilidade de Interrupção}

A potência do sinal no $i$-ésimo receptor é dada por $G_{i i} F_{i i} P_{i}$ e a potência total interferente é dada por:

$$
\sum_{k \neq i} G_{i k} F_{i k} P_{k}
$$
é dada por:

$$
S I R_{i}=\frac{G_{i i} F_{i i} P_{i}}{\sum_{k \neq i} G_{i k} F_{i k} P_{k}}
$$

Considera-se neste trabalho que a qualidade de serviço, QoS (quality of service), pode ser atingida quando a $S I R_{i}$ excede uma dada SIR de limiar, $S R^{\text {th }}$. Assim, a probabilidade de interrupção para o $i$-ésimo par receptor/transmissor é prontamente obtida:

$$
\begin{aligned}
O_{i} & =\operatorname{Prob}\left(\operatorname{SIR}_{i} \leq \operatorname{SIR}^{t h}\right) \\
& =\operatorname{Prob}\left(G_{i i} F_{i i} P_{i} \leq \operatorname{SIR}^{t h} \sum_{k \neq i} G_{i k} F_{i k} P_{k}\right)
\end{aligned}
$$

A probabilidade $O_{i}$ pode ser interpretada como a fração de tempo durante a qual o $O_{\text {-ésimo par }}$ transmissor/receptor experimenta uma quebra de comunicação devido ao efeito do desvanecimento do canal. Note-se que em (5) foi levada em consideração a variação estatística da potência do sinal recebido e da potência de interferência em um sistema de múltiplo acesso por divisão de código do tipo sequência direta (DS/CDMA).

Uma expressão analítica para $O_{i}$ foi derivada em (KANDUKURI; BOYD, 2002) partindo-se da hipótese de que $z_{1}, \ldots, z_{n}$ sejam variáveis aleatórias independentes, distribuídas exponencialmente e com médias $\mathbb{E}\left[z_{i}\right]=1 / \lambda_{i}$. Dadas essas suposições, pode-se mostrar que para um canal com desvanecimento Rayleigh resulta:

$$
\operatorname{Prob}\left(z_{1} \leq \sum_{i=2}^{n} z_{i}\right)=1-\prod_{i=2}^{n}\left(\frac{1}{1+\left(\lambda_{1} / \lambda_{i}\right)}\right)
$$

Assim, incluindo-se o efeito do ruído aditivo branco Gaussiano (AWGN) a partir da introdução de uma constante ${ }^{c}$ em (6), tem-se:

$$
\operatorname{Prob}\left(z_{1}>\sum_{i=2}^{n} z_{i}+c\right)=c^{-\lambda_{1} c} \prod_{i=2}^{n}\left(\frac{1}{1+\left(\lambda_{1} / \lambda_{i}\right)}\right)
$$

Isto pode complicar a análise do problema de otimização resultante. Desta forma, neste trabalho foi assumido nas análises que o AWGN não está 
presente, ou seja, na prática que a potência de ruído é insignificante quando comparado às potências dos sinais interferentes. Assim, aplicando em (5) o resultado obtido em (6), tem-se que a probabilidade de interrupção para o $i$-ésimo par transmissor/ receptor pode ser expressa como:

$$
O_{i}=1-\prod_{k \neq i}\left(1+S R^{t h} \frac{G_{i k} P_{k}}{G_{i i} P_{i}}\right)^{-1}
$$

Define-se como probabilidade de interrupção do sistema de comunicação de múltiplo acesso o maior valor para $O_{i}$ em (8), representando a pior probabilidade de interrupção sobre todos os pares transmissores/receptores, que pode ser expressa como:

$$
O=\max _{i} O_{i}
$$

A probabilidade de interrupção global do sistema serve como uma simples figura de mérito para a determinação da melhor política de alocação de potência.

\section{Margem de Certeza Equivalente}

Considera-se agora o conceito de certeza equivalente, no qual é ignorada toda a variação estatística da potência do ruído e do sinal, sendo substituídas estas variáveis aleatórias por suas respectivas médias estatísticas. A certeza equivalente da potência do sinal para o $i$-ésimo receptor é então $\boldsymbol{l}$ e a certeza equivalente da potência interferente para o $i$-ésimo receptor é dada por $\sum_{k \neq i} G_{i k} P_{k}$. Definese então a certeza equivalente da SIR para o $i$-ésimo receptor como (KANDUKURI; BOYD, 2002):

$$
\operatorname{SIR}_{i}^{c e}=\frac{G_{i i} P_{i}}{\sum_{k \neq i} G_{i k} P_{k}}
$$

A $S_{i}^{c e}$ pode ser interpretada como o que seria a razão sinal-interferência do $i$-ésimo par transmissor/receptor se o estado do desvanecimento do sistema fosse $F_{1}=\ldots=F_{n}=1$.
A mínima certeza equivalente do sistema sobre todos os pares transmissores/receptores, $S I R^{c e}$, pode ser definida como (KANDUKURI; BOYD, 2002):

$$
S I R^{c e}=\min _{i} S I R_{i}^{c e}=\min _{i} \frac{G_{i i} P_{i}}{\sum_{k \neq i} G_{i k} P_{k}}
$$

Faz-se referência a $S I R^{c e}$, como simplesmente, a certeza equivalente $S I R$. Da mesma forma que na probabilidade de interrupção $O$, a $S I R^{c e}$ oferece-nos uma figura de mérito alternativa para a política de alocação de potência do sistema de múltiplo acesso.

Define-se margem de certeza equivalente para o sistema, como a razão entre a certeza equivalente SIR e o limiar de recepção para a relação sinalinterferência:

$$
C E M=\frac{S I R^{c e}}{S I R^{t h}}=\min _{i} \frac{G_{i i} P_{i}}{S I R^{t h} \sum_{k \neq i} G_{i k} P_{k}}
$$

Note-se que há uma relação entre CEM $\mathrm{e}^{O}$ : quando $C E M$ cresce, o que significa que a SIR, ignorando a variação estatística do canal, esta bem acima do mínimo requerido para a recepção, o valor de $O$ é reduzido.

\section{Relação Entre a CEM e a Probabilidade de Interrupção}

Pode-se derivar alguns limites entre a $C E M$ e a probabilidade de interrupção, utilizando-se do seguinte resultado (KANDUKURI; BOYD, 2002): se $z_{1}, \ldots, z_{n} \geq 0$, então:

$$
1+\sum_{k=1}^{n} z_{k} \leq \prod_{k=1}^{n}\left(1+z_{k}\right) \leq \exp \sum_{k=1}^{n} z_{k}
$$

sendo $z_{k} \in \mathbb{R}$. Por definição, tem-se:

$$
O=\max _{i}\left(1-\prod_{k \neq i} \frac{1}{1+\frac{S I R^{t h} G_{i k} P_{k}}{G_{i i} P_{i}}}\right)=1-\frac{1}{\max _{i} \prod_{k \neq i}\left(1+\frac{S R^{t h} G_{i i} P_{k}}{G_{i i} P_{i}}\right)}
$$

Utilizando o lado direito na inequação em (13), tem-se: 


$$
O^{C} \leq 1-\frac{1}{\exp \left(\max _{i} \sum_{k \neq i} \frac{S I R^{t h} G_{i k} P_{k}}{G_{i i} P_{i}}\right)}=1-e^{-1 / C E M}
$$

De maneira similar, utilizando lado esquerdo da inequação em (13), tem-se:

$$
O \geq \frac{1}{1+\max _{i} \sum_{k \neq i} \frac{S I R^{t h} G_{i k} P_{k}}{G_{i i} P_{i}}}=\frac{1}{1+C E M}
$$

Das inequações (15) e (16), tem-se os limitantes para a probabilidade de interrupção:

$$
\frac{1}{1+C E M} \leq O \leq 1-e^{-1 / C E M}
$$

Gráficos para os limites em (17) são dados nas Figs. 1(a) e 1(c). Dos gráficos, fica claro que para a probabilidade interrupção de interesse, ou seja, aquelas menores que $20 \%$, os limites superior e inferior estão muito próximos. A Fig. 1 (b) ilustra a razão do limite superior para o limite inferior em função de $C E M$. Este gráfico mostra que os limites estão muito próximos para probabilidades de interrupção menores que 10\% e não tão distante para outros valores maiores, mesmo para $C E M$ pequena (e grande $O$ ). Por exemplo, com $C E M$ igual a um, a probabilidade de interrupção é de ao menos $50 \%$ e não mais que $63 \%$.

Figura 1 - (a) e (c) Limites superior e inferior para a probabilidade de interrupção em função da CEM, considerando amplo intervalo [1;20] e curto intervalo [0; 1] para CEM, respectivamente; (b) e (d) Razão entre o limites superior e inferior da probabilidade de interrupção em função da CEM, considerando amplo intervalo $[1 ; 20]$ e curto intervalo $[0 ; 1]$ para CEM, respectivamente

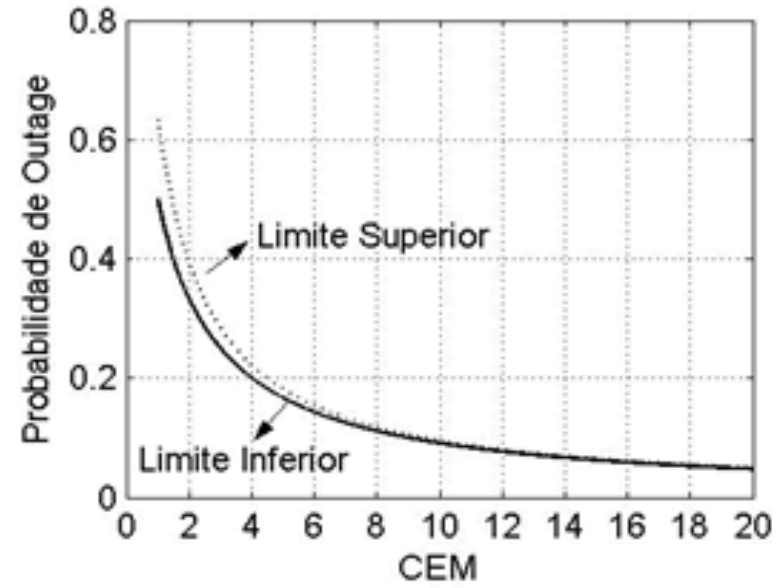

(a)

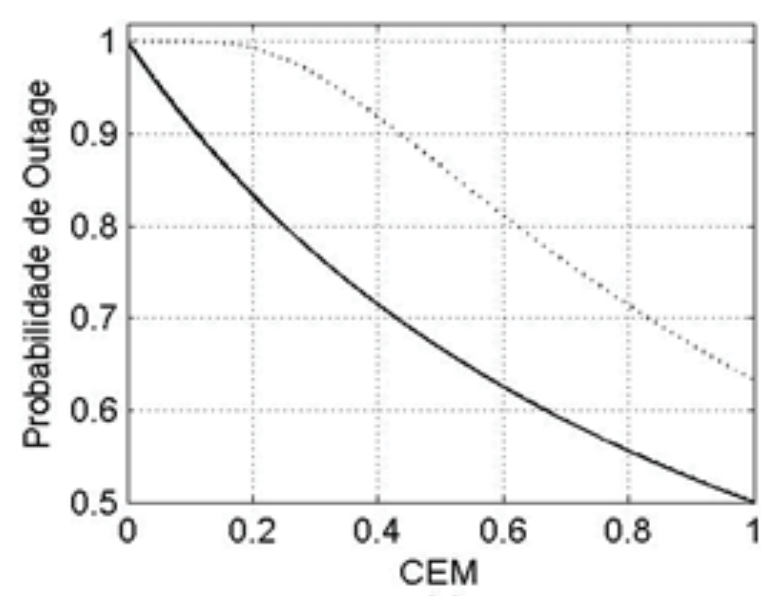

Fonte: do autor

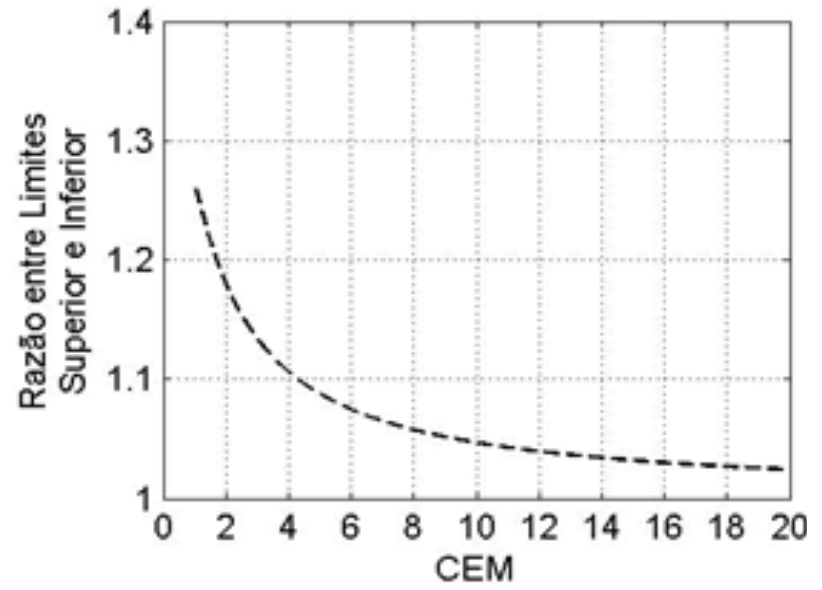

(b)

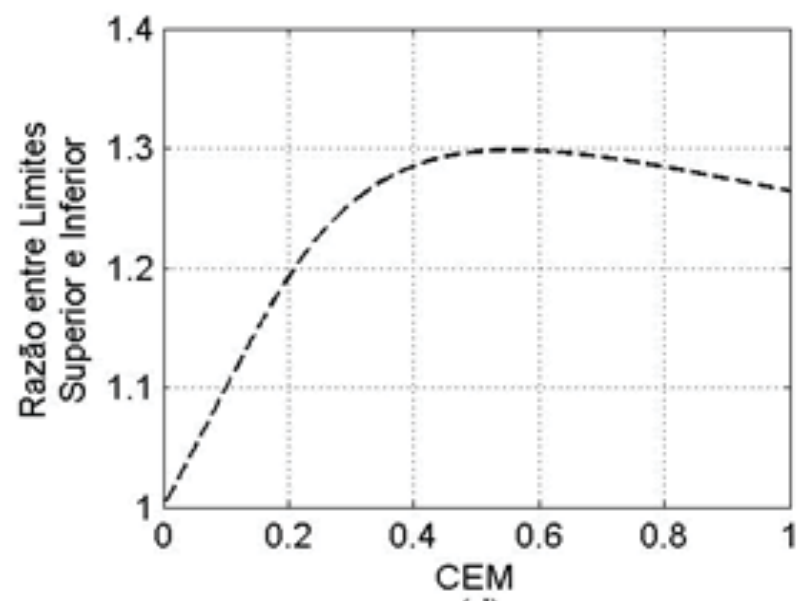

(d) 
Da Fig. 1 (d) fica evidente que há um afastamento máximo entre os limites superior e inferior, o que ocorre para um valor específico de CEM. Da Fig. 1 (d) pode-se afirmar que o limite superior não ultrapassa o inferior em mais do que 30\% para qualquer valor de $C E M$.

A razão entre os limites superior e inferior é dada por:

$$
r=(1+C E M)\left(1-e^{-1 / C E M}\right)
$$

Portanto, a $C E M$ que maximiza a razão $r$, i.e., $C E M_{r \max }$, satisfaz a seguinte equação:

$$
1+C E M_{r \max }^{-1}+C E M_{r \max }^{-2}=e^{\left(C E M_{r \text { max }}^{-1}\right)}
$$

Não é possível encontrar uma "expressão fechada" para a $C E M_{r \text { max }}$ a partir de (19). Uma forma alternativa de resolver esta equação é definir uma sequência $\left\{x_{n}\right\}$ que converge para a solução procurada. Para tanto, note-se que (19) equivale a:

$$
x=\ln \left(1+x+x^{2}\right), \text { onde } x=C E M_{r \max }^{-1}
$$

Assim, a solução de (19) pode ser obtida encontrando-se o ponto fixo da iteração:

$$
x_{n+1}=f\left(x_{n}\right)=\ln \left(1+x_{n}+x_{n}^{2}\right)
$$

Evidentemente, há inúmeras formas de se resolver (19) como um algoritmo de ponto fixo. Entretanto, poucas delas convergem, ou seja, a maioria possui ponto fixo assintoticamente instável. Neste trabalho, a forma em (21) foi a escolhida porque seu ponto fixo positivo é assintoticamente estável; vide páginas 304-308 de (MONTEIRO, 2002). Iniciando (21) com $x_{0}=1$ obtém-se uma seqüência $\left\{x_{n}\right\}$ que estabiliza no valor 1,7933 . Portanto, $C E M_{r \text { max }} \approx 0,5576$ e $r_{\text {max }} \approx 1,2984$. Note-se que estes valores estão coerentes com o gráfico da Fig. 1 (d).

\section{Alocação Ótima de Potência}

Nesta seção consideram-se os problemas de encontrar as alocações de potência que maximizam a $C E M$, minimizam as potências transmitidas e minimizam a probabilidade de interrupção. O problema de minimizar a probabilidade de interrupção pode ser expresso como:

$$
\begin{aligned}
& \text { minimizar } \quad O=\max _{\mathrm{i}}\left(1-\prod_{k \neq i} \frac{1}{1+\frac{S I R^{t h} G_{i k} P_{k}}{G_{i i} P_{i}}}\right) \\
& \text { Sujeito a: } \quad P_{i}>0, \quad i=1, \ldots, n
\end{aligned}
$$

e o problema de maximizar a $C E M$ pode ser expresso como o problema de otimização:

$$
\begin{array}{ll}
\text { maximizar } & C E M=\min _{i} \frac{G_{i i} P_{i}}{S I R^{t h} \sum_{k \neq i} G_{i k} P_{k}} \\
\text { sujeitos a: } & P_{i}>0, \quad i=1, \ldots, n
\end{array}
$$

Nestes problemas, os valores a serem encontrados são as potências $P_{1}, \ldots, P_{n}$. Assume-se que o nível de QoS definido por $S^{\text {th }}$ e os ganhos $G_{i k}, i, k=1, \ldots, n$ são parâmetros positivos conhecidos, mesmo que sujeito a algum erro de estimativa; adicionalmente, neste contexto, admite-se que os valores de potências solução para os problemas (22) e (23) atendem a $P_{i} \leq P_{\max }$, sendo $P_{\max }$ a máxima potencia de rádio frequência disponível.

Denotar-se-á por $\mathrm{P}^{\text {out }}$ um vetor de alocação de potência que é ótimo para o problema (22), isto é, que minimiza a probabilidade de interrupção. Similarmente, $\mathbf{P}^{\text {cem }}$ representará um vetor de alocação de potência que é ótimo para o problema (23), ou seja, que maximiza a $C E M$.

Cabe a observação que em cada problema, o ótimo é encontrado com os valores de máximo (para 
minimizar ${ }_{C E M}$ ) ou mínimo (para maximizar $C E M$ ) todos iguais. Considere primeiro o problema (22) de minimizar a probabilidade de interrupção. Pretende-se mostrar que, para um vetor de alocação de potência ótimo $\mathbf{P}^{\text {out }}$ as probabilidades de interrupção para cada par transmissor/receptor são iguais, ou seja:

$$
O_{i}\left(\mathbf{p}^{\text {out }}\right)=1-\prod_{k \neq i} \frac{1}{1+\frac{S I R^{\text {th }} G_{i k} P_{k}^{\text {out }}}{G_{i i} P_{i}^{\text {out }}}}=O\left(\mathbf{p}^{\text {out }}\right)=O^{*}, \quad i=1, \ldots, n
$$

sendo $O^{*}$ o valor máximo aceitável para a probabilidade de interrupção. Para se estabelecer este resultado, observa-se que a $O_{i}$ é uniformemente crescente em $P_{k}$ para $k \neq i$ e uniformemente decrescente em $P_{i}$. Agora suponha que nem todas $O_{i}$ são iguais. Escolha um indexador $k$ para o qual $O_{k}<O^{*}=\max _{i} O_{i}$. Para um decréscimo em $P_{k}^{\text {out }}, O_{k}$ incrementa e todos as outras $O_{i}$ sofrem decréscimo. Para um pequeno decréscimo em $P_{k}^{\text {out }}, O=\max _{i} O_{i}$ será decrescida. Contudo, isto contradiz a hipótese que $P^{\text {out }}$ minimiza $O$.

Resultado análogo permanece para o problema (23) de maximizar CEM. Neste problema, observa-se que cada $C E M$ é monotonicamente crescente em $P_{i}$ e monotonicamente decrescente em $P_{k}$ para $k \neq i$. Com o mesmo argumento anterior, pode-se concluir que:

$$
O_{i}\left(\mathbf{p}^{\text {out }}\right)=1-\prod_{k \neq i} \frac{1}{1+\frac{S I R^{\text {th }} G_{i k} P_{k}^{\text {out }}}{G_{i i} P_{i}^{\text {out }}}}=O\left(\mathbf{p}^{\text {out }}\right)=O^{*}, \quad i=1, \ldots, n
$$
CEM .

sendo $i=1, \ldots, n$ e $C E M^{*}$ é o máximo valor de

\section{Maximizando a CEM}

No campo de redes sem fio, o controle de potência pormaximização da $C E M$ tem sidobastante estudado (MITRA, 1993), (BAMBOS, 1998), (ALAVI; NETTLETON, 1982), (AEIN, 1973), (ZANDER, 1992), (YUN; MESSERSCHMITT, 1994), (YUN; MESSERSCHMITT, 1995), (ZANDER, 1992).
Tal problema está baseado no teorema de PerronFrobenius para o máximo autovalor de uma matriz que tem elementos não negativos.

Utilizando a observação de que para o valor ótimo, todas as $C E M$ são iguais, pode-se reformular o problema (23) como:

$$
\begin{array}{ll}
\text { maximizar } & t \\
\text { sujeito a: } & \frac{G_{i i} P_{i}}{S^{\text {th }} \sum_{k \neq i} G_{i k} P_{k}}=t, \quad i=1, \ldots, n \\
& P_{i}>0, \quad i=1, \ldots, n
\end{array}
$$

onde $t$ é outra variável, cujo valor ótimo também é o ótimo de $C E M$. Substituindo a variável $\tau=1 / t$ , o problema pode ser expresso como:

$$
\begin{array}{ll}
\text { minimizar } & \tau \\
\text { sujeito a: } & \mathbf{A P}=t \mathbf{P} \\
& P_{i}>0, \quad i=1, \ldots, n
\end{array}
$$

onde a matriz A tem seu elementos definidos por:

$$
A_{i j}=\frac{\operatorname{SIR}^{t h} G_{i j}}{G_{i i}}, \quad i \neq j, \quad A_{i i}=0 .
$$

O problema acima pode ser identificado como um problema de autovalor no qual a matriz tem todas as entradas não negativas. De acordo com a teoria de Perron-Frobenius, o autovalor $\lambda$ de $\mathbf{A}$ que é maior em magnitude, é real e positivo e tem um autovetor $\mathbf{V}$ associado, cujos componentes são positivos. O autovetor $\mathbf{V}$ (e o autovalor associado $\lambda$ ) são chamados de autovetor (autovalor) de Perron-Frobenius de A. O autovetor de PerronFrobenius $\mathbf{V}$ resulta em uma alocação de potência ótima, isto é, $\mathbf{P}=\mathbf{v}$ maximiza a $C E M$. A $C E M$ ótima é exatamente $C E M \quad /$. 


\section{Relação Entre Alocação De CEM Ótima e $O$} Ótimo

Utilizando os limites encontrados na seção anterior, pode-se mostrar que uma alocação de potência que maximiza a $C E M$, a qual pode ser encontrada pelo cálculo do autovetor PerronFrobenius de uma matriz $n \times n$, está muito próxima da minimização da probabilidade de interrupção.

Denotando por $\mathbf{P}$ uma alocação de potência arbitrária $\left(\operatorname{com} P_{i}>0\right)$, tem-se:

$$
C E M(\mathbf{P}) \leq C E M\left(\mathbf{P}^{\mathrm{cem}}\right)
$$

uma vez que, por definição, $\mathbf{P}^{\text {cem }}$ maximiza a $C E M$. Segue que:

$$
\frac{1}{1+C E M(\mathbf{P})} \geq \frac{1}{1+C E M\left(\mathbf{P}^{\mathrm{cem}}\right)}
$$

uma vez que a função de mapeamento de $1 /(1+x)$ é monotonicamente decrescente para $x>0$. Combinando esta inequação com o lado esquerdo em (17), tem-se:

$$
O(\mathbf{P}) \geq \frac{1}{1+C E M(\mathbf{P})} \geq \frac{1}{1+C E M\left(\mathbf{P}^{\mathrm{cem}}\right)}
$$

Esta inequação é válida para qualquer $\mathbf{P}$, assim:

$$
O^{*} \geq \frac{1}{1+C E M\left(\mathbf{P}^{\mathrm{cem}}\right)}
$$

onde $O^{*}$ denota a probabilidade de interrupção mínima possível, isto é, o valor ótimo para o problema (22). Desta inequação, pode-se tirar algumas conclusões: através do cálculo de $\mathrm{P}^{\mathrm{cem}}$ (resolvendo um problema de autovetor de PerronFrobenius), pode-se obter fronteiras para $O^{*}$, pois certamente este estará entre o limite inferior $\left(1+C E M\left(\mathbf{P}^{\mathrm{cem}}\right)\right)^{-1}$ e o limite superior $O\left(\mathbf{P}^{\mathrm{cem}}\right)$ . Estes limites estão situados, com freqüência, extremamente próximos. Uma vez que:

$$
O\left(\mathbf{P}^{\mathrm{cem}}\right) \leq 1-e^{-1 / C E M}
$$

ter-se-á:

$$
\frac{1}{1+C E M\left(\mathbf{P}^{\mathrm{cem}}\right)} \leq O^{*} \leq 1-e^{-1 / \operatorname{CEM}\left(\mathbf{P}^{\mathrm{cem}}\right)}
$$

Uma vez que a razão destes limites está com freqüência próxima de um e nunca distante de um, segue que o resultado para maximização da $C E M$ está com freqüência muito próximo do resultado de minimizar a probabilidade de interrupção.

\section{Minimizando a Potência de Transmissão}

Nesta seção, considera-se o problema de minimizar a potência total transmitida sujeita a restrições de interrupção ou $C E M$ e limites sobre potências individuais. Será mostrado que o problema de alocação de potência com restrições sobre a probabilidade de interrupção, bem como outras restrições, tais como limites sobre as potências individuais, pode ser expresso como um tipo especial de problema de otimização conhecido como programação geométrica (DUFFIN; PETERSON; ZENER, 1967), (BOYD; VANDENBERGHE, 2005), (CHIANG, 2005).

Para minimizar a potência total transmitida, com a restrição de que cada par transmissor/receptor atinge uma probabilidade de interrupção máxima e com limites sobre as potências transmitidas, formula-se o problema da seguinte forma:

$$
\begin{array}{lll}
\text { minimizar } & P_{1}+\ldots+P_{n} \\
\text { Sujeito a } & P^{\min } \leq P_{i} \leq P_{i}^{\max }, & i=1, \ldots, n \\
& O_{i} \leq O_{i}^{\max }, & i=1, \ldots, n
\end{array}
$$

Aqui, $P_{i}^{\min }$ e $O_{i}^{\max }$ são a mínima e a máxima potência transmitida no i-ésimo uplink (UL) do sistema de múltiplo acesso CDMA; a máxima 
potência pode ser dependente do hardware do transmissor e o mínimo valor garante que o ruído branco no receptor seja superado. $\mathrm{O}$ valor $O_{i}^{\max }$ define a máxima probabilidade de interrupção tolerável para o $i$-ésimo par transmissor/receptor, sendo uma medida de QoS.

Utilizando (8), pode-se expressar a restrição probabilidade de interrupção $O_{i} \leq O_{i}^{\max }$ como:

$$
\begin{gathered}
1-O_{i}^{\mathrm{max}} \leq \prod_{k \neq i}\left(\frac{1}{1+\frac{S I R^{t h} G_{i k} P_{k}}{G_{i i} P_{i}}}\right) \\
\left(1-O_{i}^{\max }\right) \prod_{k \neq i}\left(1+\frac{S I R^{t h} G_{i k} P_{k}}{G_{i i} P_{i}}\right) \leq 1
\end{gathered}
$$

Umavez que cada um dostermos $1+\left(\operatorname{SIR}^{\text {th }} G_{i k} P_{k} / G_{i i} P_{i}\right)$ é uma função posynomial ${ }^{3}$ (CHIANG, 2005) de potências, conclui-se que o lado esquerdo da inequação (37) é, de fato, uma função posynomial de potências $P_{1}, \ldots, P_{n}$.

Utilizando este resultado, pode-se expressar o problema (35) agora como:

$$
\begin{array}{ll}
\text { minimizar } & P_{1}+\ldots+P_{n} \\
\text { Sujeito a } & \frac{P_{i}^{\min }}{P_{i}} \leq 1, \quad i=1, \ldots, n \\
& \frac{P_{i}}{P_{i}^{\max }} \leq 1, \quad i=1, \ldots, n \\
& \left(1-O_{i}^{\max }\right) \prod_{k \neq i}\left(1+\frac{S I R^{t h} G_{i k} P_{k}}{G_{i i} P_{i}}\right) \leq 1
\end{array}
$$

Este é um programa geométrico (GP) nas variáveis $P_{1}, \ldots, P_{n}$. Note-se que quaisquer outras restrições que possam ser modeladas por programação geométrica podem se adicionadas ao problema de alocação de potência original.

\section{Probabilidade de Interrupção Mínima}

A formulação do problema (38) pode ser levemente modificada para minimizar a probabilidade de interrupção $O$. Neste caso, devese resolver o programa geométrico da equação (39) com variáveis de otimização $P_{1}, \ldots, P_{n}$ e $\alpha$. Aqui, $\alpha$ é um limite superior sobre $1 /\left(1-O_{i}^{\max }\right)$ e, portanto, quando se resolve o GP (39), o valor ótimo de $\alpha$ deve ser $1 /\left(1-O^{*}\right)$, onde $O^{*}$ é o valor mínimo da probabilidade de interrupção máxima. Ainda que GPs possam ser resolvidos, eles tomam um tempo considerável comparado às exigências de rapidez de processamento de sinal no receptor do sistema de comunicação.

$$
\begin{array}{ll}
\text { minimizar } & \alpha \\
\text { Sujeito a } & \frac{P_{i}^{\min }}{P_{i}} \leq 1, \quad i=1, \ldots, n \\
& \frac{P_{i}}{P_{i}^{\max }} \leq 1, \quad i=1, \ldots, n \\
& \left(\frac{1}{\dot{a}}\right) \prod_{k \neq i}\left(1+\frac{S I R^{t h} G_{i k} P_{k}}{G_{i i} P_{i}}\right) \leq 1
\end{array}
$$

Uma metodologia de rápida convergência, baseada no teorema de Perron-Frobenius, para minimizar probabilidade de interrupção sem qualquer restrição de mínimo/máximo sobre as potências foi proposto em (KANDUKURI; BOYD, 2002). Como todas as probabilidades de interrupção são iguais na condição de mínimo valor, o problema (39) pode ser reescrito da seguinte forma:

$$
\begin{array}{ll}
\text { minimizar } & t \\
\text { Sujeito a } & 1-\prod_{k \neq i} \frac{1}{1+\frac{S I R^{t h} G_{i k} P_{k}}{G_{i i} P_{i}}}=t, \quad i=1, \ldots, n \\
& P_{i}>0, \quad i=1, \ldots, n
\end{array}
$$

O algoritmo consiste em resolver um problema de autovetor de Perron-Frobenius em cada iteração. Não há uma prova de que o método sempre converge, mas de acordo com (KANDUKURI; BOYD, 2002) a convergência se dá em no máximo cinco iterações. Os cinco passos a seguir descrevem o método de Kandukuri e Boyd.

\footnotetext{
${ }^{3}$ Uma função $a_{i} \in \mathbb{R}$ com domínio $\operatorname{dom} f=\mathrm{R}^{\wedge}{ }_{-}\{++\}$definida como $f(x)=c \cdot x_{1}^{a_{1}} \cdot x_{2}^{a_{2}} \cdots x_{n}^{a_{n}}$, sendo $c>0$ e $a_{i} \in \mathbb{R}$ é denominada função monomial. A soma de funções monomiais, $f(x)=\sum_{k=1}^{K} c_{k} \cdot x_{1}^{a_{1 k}} \cdot x_{2}^{a_{2 k}} \cdots x_{n}^{a_{n k}}$ com $c_{k}>0$ é denominada função posynomial.
} 
1. Inicia-se com as restrições de igualdade:

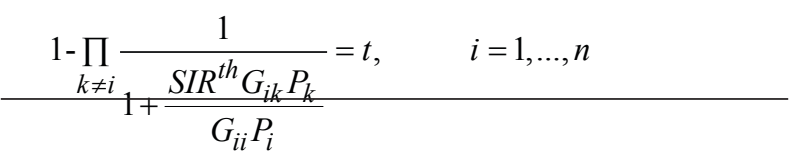

sendo $t$ uma variável a ser minimizada. Esta equação pode ser reescrita como:

$$
\prod_{k \neq i}\left(1+\frac{S I R^{t h} G_{i k} P_{k}}{G_{i i} P_{i}}\right)=\beta, \quad i=1, \ldots, n
$$

$\operatorname{com} \beta=1 /(1-t)$. Aqui, o objetivo é minimizar $\beta$. Pode-se reescrever estas equações na formal(43)

$$
\sum_{k \neq i} \log \left(1+\frac{S I R^{t h} G_{i k} P_{k}}{G_{i i} P_{i}}\right)=\gamma, \quad i=1, \ldots, n
$$

sendo $\gamma=\log \beta$ o objetivo a ser minimizado. Isto é equivalente a:

$$
\sum_{k \neq i}\left[\frac{P_{i}}{P_{k}} \log \left(1+\frac{S I R^{t h} G_{i k} P_{k}}{G_{i i} P_{i}}\right)\right] P_{k}=\gamma P_{i}
$$

a qual pode ser expressa como $\mathbf{B}(\mathbf{P}) \mathbf{P}=g \mathbf{P}$, onde $\mathbf{B}$ é uma matriz, cujos elementos são dados por: ${ }^{(4)}$

$$
B_{i k}=\frac{P_{i}}{P_{k}} \log \left(1+\frac{S I R^{t h} G_{i k} P_{k}}{G_{i i} P_{i}}\right), \quad i \neq k
$$

e $B_{i i}=0$.

2. Encontre o vetor $\mathbf{P}$ com entradas positivas que minimiza $\gamma$ e satisfaz a condição $\mathbf{B}(\mathbf{P}) \mathbf{P}=\gamma \mathbf{P}$. Se for ignorado o fato de que $\mathbf{B}$ depende de $\mathbf{P}$, este problema pode ser resolvido como um problema de autovetor de Perron-Frobenius.

$$
\text { 3. Inicia-se o método iterativo com } \mathbf{P}=\mathbf{P}^{\text {cem }}
$$

4. Fixa-se $\mathbf{B}=\mathbf{B}(\mathbf{P})$ e atualiza-se $\mathbf{P}$ pela resolução do problema de autovetor de Perron-Frobenius $\mathbf{B P}=\gamma \mathbf{P}$.

5. Isto é repetido até que elementos de $\mathbf{P}$ não sofram alterações na iteração seguinte.

\section{Implementações}

A partir do simulador matemático MATLAB v.7.0 implementou-se um algoritmo para resolver o problema da CEM ótimo, dado em (27). Considerou-se um sistema com 50 pares transmissor/ receptor submetidos a desvanecimentos Rayleigh/ Rayleigh, sendo a potência do AWGN considerada desprezível quando comparada à potência dos sinais interferentes.

\section{Maximizando a $C E M$}

Uma vez que a matriz de ganhos $G_{i j}$ é o produto de diferentes quantidades aleatórias variantes no tempo, pode-se simplificar o problema a partir de dos seguintes passos:

1. Toma-se todos os ganhos $G_{i i}$ (do $i$-ésimo transmissor para o $i$-ésimo receptor) como sendo um e gera-se os ganhos cruzados $G_{i j}, i \neq j$, como variáveis aleatórias independentes e distribuídas uniformemente entre zero e $0,001^{4}$.

2. Varia-se $S I R^{\text {th }}$ de 1 a 10 e para cada valor calcula-se $\mathbf{P}^{\text {cem }}$ utilizando o método de maximização da $C E M$. Para cada valor de $S I R^{\text {th }}$, também calculase $O\left(\mathbf{P}^{\mathrm{cem}}\right)$, a probabilidade de interrupção alcançada por $\mathbf{P}^{\mathrm{cem}}$, bem como o limite inferior e superior, os quais estão ilustrados na Fig. 2 para diferentes valores de $S I R^{t h}$.

\footnotetext{
${ }^{4}$ Representando, respectivamente, a condição de ortogonalidade entre usuários (interferência de múltiplo acesso igual a zero) e uma condição de correlação cruzada mediana, incluindo ainda o efeito da atenuação do sinal do usuário interferente devido à distância.
} 
Figura 2 - Probabilidade de Interrupção (calculada em $\left.\mathrm{P}^{\mathrm{cem}}\right) \times \mathrm{SIR}^{\text {th }}$; sistema com 50 links

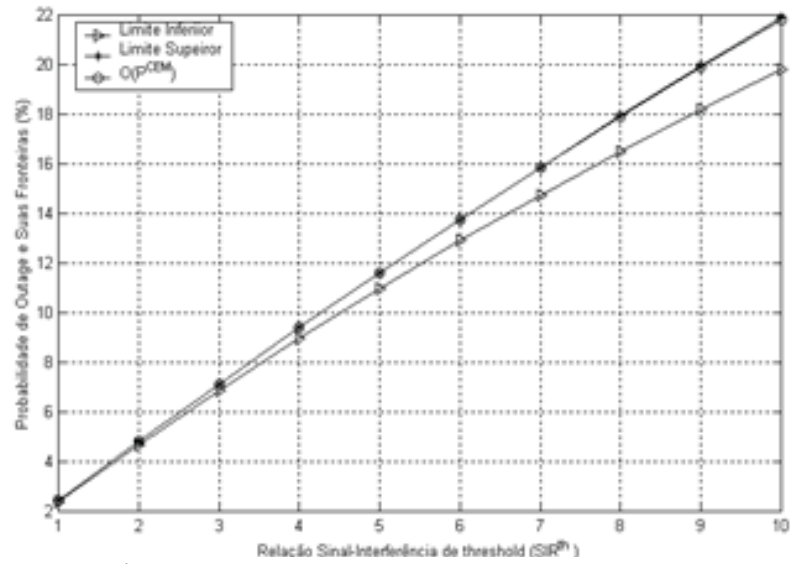

Fonte: do autor

A Fig. 3 ilustra os valores encontrados para alocação de potência ótima na maximização da CEM.

Figura 3 - Alocação de potência ótima para maximizar CEM; sistema com 50 links.

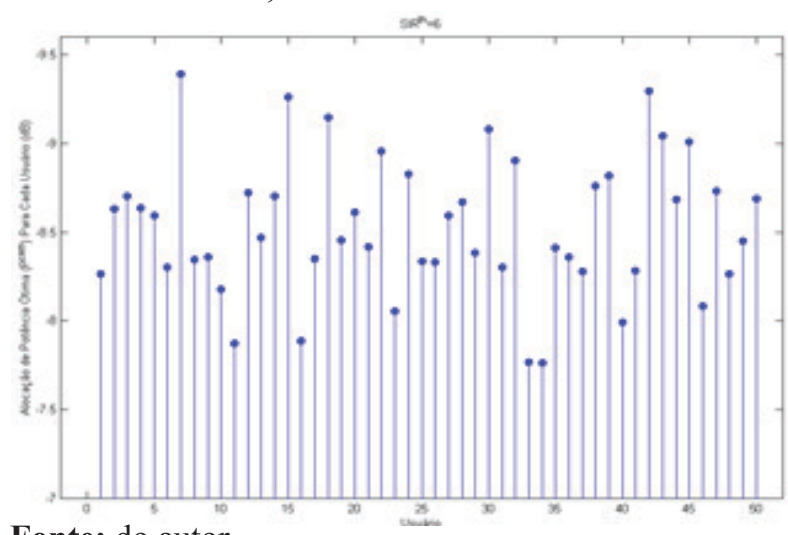

Fonte: do autor

O resultado da Fig.2 está condizente com os valores de potência alocada apresentados na Fig. 3. Note-se que as curvas de probabilidade de interrupção (calculada em $\mathrm{P}^{\text {cem }}$ ) e de limite superior praticamente se sobrepõem.

\section{Minimizando A Probabilidade De Interrupção}

Utilizando o mesmo procedimento descrito na subseção anterior "MAXIMIZANDO A CEM" para o cálculo da matriz de ganho e número de usuários, implementou-se um algoritmo no MATLAB para resolver, pelo método iterativo, o problema de otimização apresentado em (39). O vetor de potência ótimo $\mathbf{P}^{\text {out }}$ encontrado foi utilizado para calcular a probabilidade de interrupção através de (8). Definiu-se o seguinte critério de parada para o algoritmo como sendo:

$$
\max _{i}\left|\frac{P_{i_{k-1}}^{\text {out }}-P_{i_{k}}^{\text {out }}}{P_{i_{k-1}}^{\text {out }}}\right| \leq E ; \quad i=1, \ldots, n
$$

sendo o número de iterações e ${ }^{n}$ o número de links do sistema. Verificou-se que para 50 links e uma diferença entre iterações normalizada $E=0,00001$, o algoritmo executa apenas 3 a 4 iterações. Isto mostra que a convergência do algoritmo é realmente rápida (KANDUKURI; BOYD, 2002).

A Fig. 4 ilustra a probabilidade de interrupção calculada substituindo $\mathrm{P}^{\text {out }}$ e $\mathrm{P}^{\text {cem }}$ em (8), assim como os limites inferior e superior para probabilidade de interrupção, dadas por (17). Nota-se que as soluções de maximizar a CEM e minimizar a soma das potências transmitidas estão bem próximas.

Foi verificado que, neste caso, a probabilidade de interrupção calculada por $\mathbf{P}^{\text {out }}$ ou $\mathbf{P}^{\text {cem }}$ é a mesma para todos os usuários, conforme mencionado anteriormente.

Figura 4 - Probabilidade de Interrupção (calculada em $\mathrm{P}^{\text {out }}$ e $\left.\mathrm{P}^{\text {cem }}\right)$ versus $\mathrm{SIR}^{\text {th }}$ para um sistema com 50 links

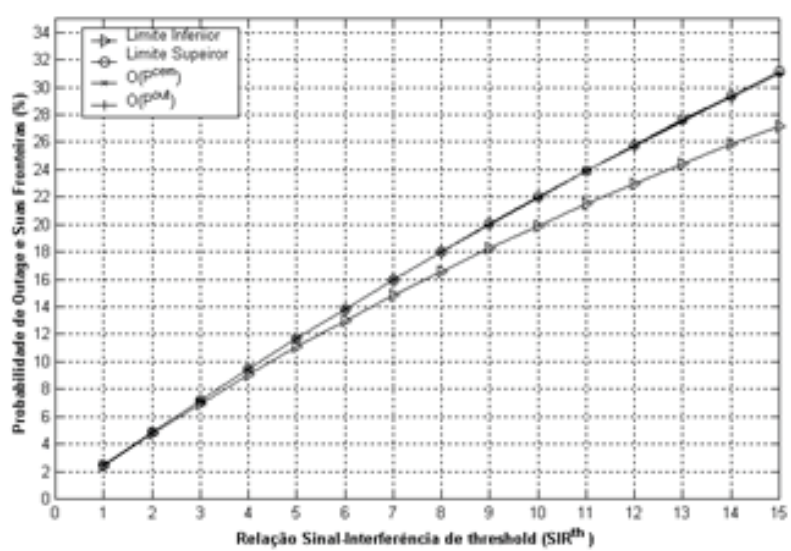


Relação entre Probabilidade de Interrupção, CEM e SIR ${ }^{\text {th }}$

Para verificar relações entre probabilidade de interrupção, CEM e $S I R^{\text {th }}$ foi realizada uma simulação semelhante à subseção anterior, porém com o número de links do sistema variando de 10 a 50, anotando-se os respectivos valores de CEM e probabilidade de interrupção. O resultado é semelhante ao obtido na Fig. 4 para probabilidade de interrupção, porém com diversos valores de $C E M$, conforme ilustrado na Fig. 5. Observase que a probabilidade de interrupção aumenta com o aumento da $S I R^{\text {th }}$, conforme ilustrado também na Fig. 2. Adicionalmente, observa-se que a probabilidade de interrupção diminui com o aumento da $C E M$, como ilustrado na Fig. 1. Nota-se ainda que a $C E M$ diminui com o aumento de $S I R^{\text {th }}$, o que está de acordo com a própria definição de $C E M$. Também pode ser observado que a probabilidade de interrupção aumenta com o incremento do número de links no sistema. Isto se deve ao fato que, com o aumento do número de links, há também um aumento da potência total dos sinais interferentes.

Figura 5 - Relações entre probabilidade de interrupção, CEM, número de links do sistema e $\mathrm{SIR}^{\text {th }}$

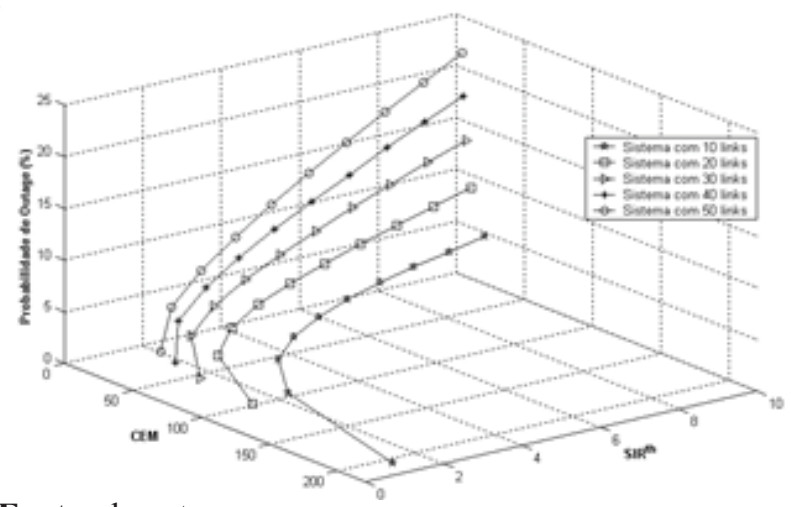

Fonte: do autor otimização não-linear (NLP) convexo, i.e., com função objetivo e restrições convexos:

$$
\begin{array}{ll}
\text { minimizar } & \ln \left(\sum_{i=1}^{n} e^{Y_{i}}\right) \\
\text { Sujeito a } & \ln \left(P_{i}^{\min }\right)-Y_{i} \leq 0, \quad i=1, \ldots, n \\
& Y_{i}-\ln \left(P_{i}^{\max }\right) \leq 0, \quad i=1, \ldots, n \\
& \ln \left(1-\mathrm{O}_{\mathrm{i}}^{\max }\right)+\sum_{k \neq i} \ln \left(1+\frac{\operatorname{SIR}^{t h} G_{i k} e^{\left(Y_{k}-Y_{i}\right)}}{G_{i i}}\right) \leq 0
\end{array}
$$

sendo $Y_{i}=\ln P_{i}$.

O NLP convexo (46) foi resolvido utilizando a função "fmincon" do MATLAB. Neste problema, poder-se-ia ter diferentes probabilidades de interrupção para cada usuário, assim como diferentes limites de potência transmitida por usuário. Porém, para efeito de comparação, resolveu-se o problema dado em (46) da seguinte forma: para uma matriz de ganho $G$ (utilizou-se a mesma matriz $G$ da seção 5.2), mesma probabilidade de interrupção para todos os links (sendo igual a probabilidade obtida na seção 5.2), limites mínimo e máximo de potência dados a partir do exemplo da seção 5.2 e $S I R^{\text {th }}=5$; calculou-se o vetor ótimo de alocação de potência através do algoritmo implementado. Os respectivos valores de erro relativo percentual são apresentados na Fig. 6.

Figura 6 - Erro relativo percentual entre os vetores ótimos de potência relativo às subseções "minimizando a probabilidade de interrupção" e "relação entre probabilidade de interrupção, CEM E SIR $^{\mathrm{TH}}$ " considernado 50 links.

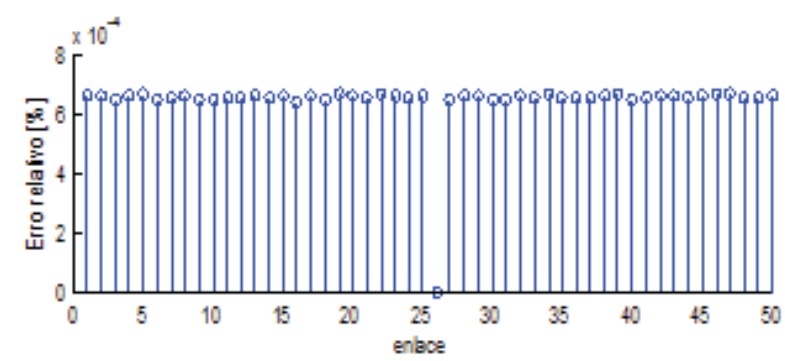

Fonte: do autor

Observa-se que os resultados do erro relativo Minimizando A Soma das Potências Transmitidas percentual entre os dois métodos estão muito próximos de zero para todos os 50 links. Desta

Por fim, o GP apresentado em (38) pode ser transformado no seguinte problema de forma, confirmou-se a validade do método iterativo de (KANDUKURI; BOYD, 2002). 


\section{Conclusões}

As implementações propostas apresentaram resultados consistentes para o problema do controle de potência em sistemas de comunicação de múltiplo acesso CDMA. Comprovou-se, por exemplo, que o método iterativo baseado na teoria de autovalores de Perron-Frobenius possui convergência rápida (em aproximadamente 4 iterações obteve-se convergência para o valor ótimo), o que torna tal metodologia de grande valor prático, pois em condições realistas de atualização do vetor de potência, a alocação deve ser atualizada 1600 vezes por segundo em sistemas de comunicação CDMA de terceira geração.

O fato do problema de alocação de potência poder ser transformado em um problema de otimização convexa não-linear permite garantir que a solução ótima encontrada é global. Assim, por exemplo, pode-se garantir que a solução encontrada, via simulação computacional, para o problema (46) é a solução ótima global (e não apenas local).

Adicionalmente, a solução do NLP convexo em (46) foi comparada com a solução obtida via método iterativo de Perron-Frobenius. Como se tem certeza que a solução de (46) leva ao mínimo global, podese dizer, pelos resultados do erro relativo percentual da Fig. 6, que o método iterativo analisado também apresentou resultados consistentes e promissores.

Confirmou-se, ainda, que a alocação de potência que maximiza a $C E M$, resolvida utilizando PerronFrobenius não-iterativo, fornece um vetor de potências muito próximo daquele vetor que se obtém pela minimização da probabilidade de interrupção.

\section{Referências}

AEIN, J. Power balancing in systems employing frequency reuse. COMSAT Tech. Rev., v. 3, n. 2, p. 277-300, 1973.

ALAVI, H.; NETTLETON, R. Power control for a spread spectrum cellular mobile radio system. In: VTC'83 - 33rd IEEE Vehicular Technology Conference, 1983, Toronto, Ontario. Anais... IEEE Publisher. 1983, v. 3, p. 242 - 246. DOI: 10.1109/ VTC.1983.1623141.
ANDERSIN, M.; ROSBERG, Z.; ZANDER, J. Gradual removals in cellular PCS with constrained power control and noise. Wireless Netw., v. 2, n. 1, p. 27-43, 1996.

ARIYAVISITAKUL, S. SIR based power control in a CDMA system. In: PROC. GLOBECOM: IEEE Global Telecommunications Conference, 1992. Orlando, Florida. Anais... Piscataway, New Jersey: IEEE Publisher, 1992. v. 2, p. 868-873.

BAMBOS, N. Toward power-sensitive network architectures in wireless communications: Concepts, issues and design aspects. IEEE Pers. Commun., v. 5, p. 50-59, Jun. 1998.

BAMBOS, N.; CHEN, S.; POTTIE, G. Radio link admission algorithms for wireless networks with power control and active link quality protection. In: PROC. IEEE INFOCOM, 1995, Boston, USA. Anais... Piscataway, New Jersey: IEEE Publisher,, 1995. v. 1, p. 97-104. DOI: 10.1109/INFCOM.1995.515865

BOYD, S.; VANDENBERGHE, L. Convex Optimization. Cambridge University Press, Cambridge, UK, 2005. Disponível em: www.convexoptimization.com Acesso em: 3 jun. 2015.

CHIANG, M. Geometric programming for communication systems. Boston, USA: Now Publishers Inc., 2005.

DUFFIN, R. J.; PETERSON, E. L.; ZENER, C. Geometric programming: theory and applications. New York: Wiley, 1967.

FOSCHINI, G.; MILJANIC, Z. A simple distributed autonomous power control algorithm and its convergence. IEEE Trans. Veh. Technol., v. 42, p. 641646, Nov. 1993. DOI: 10.1109/25.26074

FOSCHINI, G.; MILJANIC, Z. Distributed autonomous wireless channel assignment with power control. IEEE Trans. Veh. Technol., v. 44, p. 420-429, Aug. 1995.

GOLDSMITH, A.; GREENSTEIN, L.; FOSCHINI, G. Error statistics of real-time power measurements in cellular channels with multipath and shadowing. IEEE Trans. Veh. Technol., v. 43, p. 439-446, Aug. 1994. 
KANDUKURI, S.; BOYD, S. Optimal power control in interference-limited fading wireless channels with outage-probability specifications. IEEE Trans. on Wireless Communications, v. 1, n. 1, p. 46-55, Jan. 2002. DOI: 10.1109/7693.975444

MITRA, D. An asynchronous distributed algorithm for power control in cellular radio systems. In: PROC. , $4^{\text {th }}$ WINLAB WORKSHOP, 1993, Oct. 1920. Anais... New Jersey, USA, 1993. p. 249-257.

MONTEIRO, L. H. A. Sistemas dinâmicos. São Paulo: Livraria da Física, 2002.

STUBER, G. Principles of Mobile Communication. Norwell, MA: Kluwer, 1997.

YATES, R. A framework for uplink power control in cellular radio systems. IEEE J. Select. Areas Commun., v. 13, p. 1341-1347, Jul. 1995.

YATES, R.; HUANG, C. Y. Integrated power control and base station assignment. IEEE Trans. Veh. Technol., v. 44, p. 638-644, Aug. 1995.

YUN, L. C.; MESSERSCHMITT, D. G. Va riable quality of service in CDMA systems by statistical power control. In: ICC'95 - PROC. IEEE INT. CONF. COMMUNICATIONS, 1995, Seattle Anais... Piscataway, NewJersey: IEEE Publisher, 1995. v. 2, p. 713-719. DOI: 10.1109/ICC.1995.524197

YUN, L. C.; MESSERSCHMITT, D. G. Power control for variable QoS on a CDMA channel. In: PROC. IEEE MILCOM, 1994, Fort Monmouth, NJ, USA. Anais... Piscataway, New Jersey: IEEE Publisher, 1994. v. 1, p. 178-182.

ZANDER, J. Distributed cochannel interference control in cellular radio systems. IEEE Trans. Veh. Technol., v. 41, p. 305-311, Aug. 1992.

ZANDER, J. Performance of optimum transmitter power control in cellular radio systems. IEEE Trans. Veh. Technol., v. 41, p. 57-62, Feb. 1992.

Recebido em 4 Agosto, 2014 - Received on August 4, 2014 Aceito em 15 Junho, 2015 - Accepted on June 15, 2015 
